\title{
Physical conditions in CaFe interstellar clouds
}

\author{
P. Gnaciński and M. Krogulec
}

\author{
Institute of Theoretical Physics and Astrophysics, University of Gdańsk, ul. Wita Stwosza 57, 80-952 Gdańsk, Poland \\ e-mail: pg@iftia.univ.gda.pl; fizmkr@univ.gda.pl
}

Received 3 September 2007 / Accepted 25 October 2007

\section{ABSTRACT}

Interstellar clouds that exhibit strong $\mathrm{Ca}$ I and $\mathrm{Fe}$ I lines are called $\mathrm{CaFe}$ clouds. Ionisation equilibrium equations were used to model the column densities of $\mathrm{Ca}$ II, $\mathrm{Ca}$ I, K I, Na I, Fe I and Ti II in CaFe clouds. We find that the chemical composition of CaFe clouds is solar and that there is no depletion into dust grains. CaFe clouds have high electron densities, $n_{\mathrm{e}} \approx 1 \mathrm{~cm}^{-3}$, that lead to high column densities of neutral $\mathrm{Ca}$ and $\mathrm{Fe}$.

Key words. ISM: clouds - ISM: abundances - ISM: atoms - ultraviolet: ISM

\section{Introduction}

Bondar et al. (2007) discovered a new type of interstellar cloud, so-called $\mathrm{CaFe}$ cloud. These clouds are characterised by unusually strong lines of neutral $\mathrm{Ca}$ and $\mathrm{Fe}$. The anomaly may be caused by (1) different chemical composition or (2) physical conditions in the $\mathrm{CaFe}$ clouds. Interstellar spectra of the two clouds with a very prominant $\mathrm{CaFe}$ feature are seen toward HD 90177 and HD 94910, as shown in Figs. 1 and 2. Other observations of $\mathrm{CaFe}$ clouds presented by Bondar et al. (2007) have very weak atomic lines and the lines of $\mathrm{CaFe}$ clouds may be blended with lines of common clouds.

The Solar System composition is commonly used as a reference for the chemical composition of matter. We have used the Solar System Abundances $(S S A(X)=\log X / \mathrm{H}+12)$ as summarised by Grevesse \& Sauval (2000). The deficit of elements in comparison to SSA is usually attributed to depletion onto dust grains.

\section{Column densities}

We have analysed interstellar clouds in the directions of HD 90177 and HD 94910, which are the best examples of CaFe clouds. In these two directions the $\mathrm{CaFe}$ clouds are well separated in the velocity scale from other interstellar clouds. For comparison we have used spectra of three stars with ordinary clouds: HD 76341, HD 155806 and HD 163800. The lines of sight to HD 94910, HD 76314, HD 155806 and HD 163800 were also analysed by Hunter et al. (2006).

We have used publicly available spectra obtained with the UVES spectrograph at Paranal/Chile. The spectra were obtained as part of the "Library of High - Resolution Spectra of Stars across the Hertzsprung - Russell Diagram"1 - see Bagnulo et al. (2003) for details. The spectral resolution $R=$ $\lambda / \Delta \lambda=80000$ and the spectra cover a broad range of wavelengths 3040-10400 A. We have analysed interstellar absorption lines of neutral and ionised elements: Ca I, Ca II, Fe I, K I, Na I and Ti II.

The column densities of these elements were derived for individual clouds using the profile fitting technique. The

\footnotetext{
${ }^{1}$ http://www.sc.eso.org/santiago/uvespop/
}

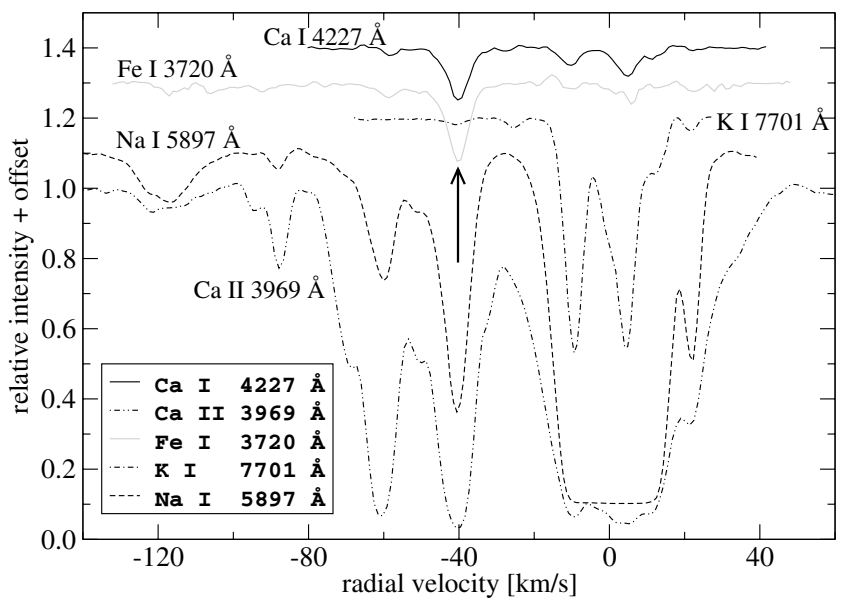

Fig. 1. Interstellar absorption lines in the spectrum of HD 90177. The arrow shows the position of the $\mathrm{CaFe}$ interstellar cloud.

absorption lines were fitted by Voigt profiles. The cloud velocities $(v)$, Doppler broadening parameters $(b)$ and column densities $(N)$ for multiple absorption components were simultaneously fitted to the observed spectrum. The lines of the Na doublet (at $5892 \AA$ and $5898 \AA$ ) were also fitted simultaneously $-v, b$ and $N$ were common for both lines in the doublet. The wavelengths, oscillator strengths $(f)$ and natural damping constants $(\Gamma)$ were adopted from Morton (2003). The derived column densities are presented in Table 1.

\section{Ionisation equilibrium}

We have used the ionisation equilibrium equation for the $\mathrm{Ca}$, $\mathrm{Fe}, \mathrm{Na}, \mathrm{K}$ and $\mathrm{Ti}$ elements to determine the physical conditions (electron density and temperature) in CaFe clouds. In the case of $\mathrm{Ca}$ the equation of ionisation equilibrium is:

$\frac{n_{\mathrm{e}} N(\mathrm{Ca} I \mathrm{II})}{N(\mathrm{Ca})}=\frac{\Gamma\left(\mathrm{Ca}_{12}\right)+n_{\mathrm{e}} C\left(\mathrm{Ca}_{12}\right)}{\alpha_{\mathrm{rad}}\left(\mathrm{Ca}_{21}\right)+\alpha_{\mathrm{die}}\left(\mathrm{Ca}_{21}\right)}$

where $N(\mathrm{Ca} I)$ and $N(\mathrm{CaI})\left[\mathrm{cm}^{-2}\right]$ are the column densities of ionised and neutral $\mathrm{Ca}, \alpha_{\mathrm{rad}}\left(\mathrm{Ca}_{21}\right)\left[\mathrm{cm}^{3} \mathrm{~s}^{-1}\right]$ is the radiative recombination coefficient, $\alpha_{\text {die }}\left(\mathrm{Ca}_{21}\right)\left[\mathrm{cm}^{3} \mathrm{~s}^{-1}\right]$ is the dielectronic 


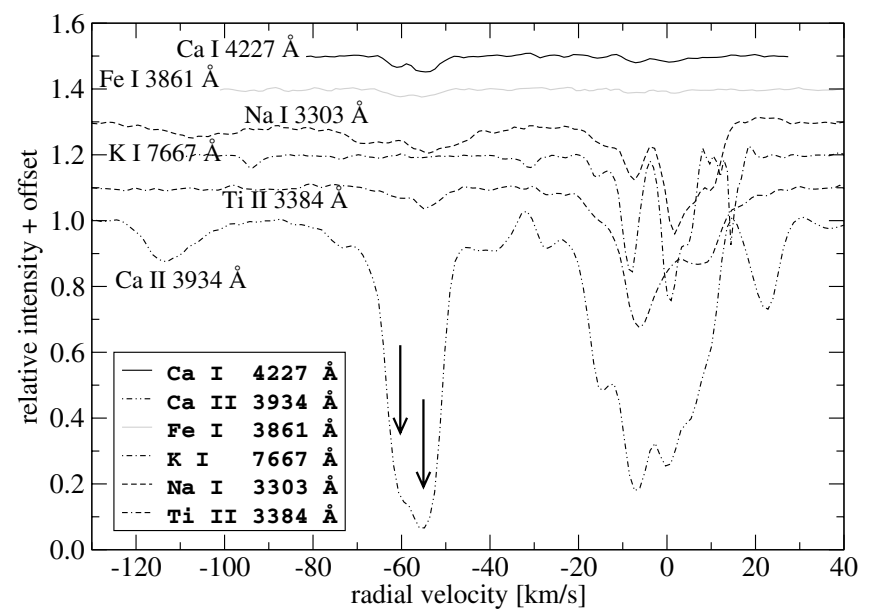

Fig. 2. Interstellar absorption lines in the spectrum of HD 94910. The arrows shows the positions of the $\mathrm{CaFe}$ interstellar clouds.

recombination rate, $\Gamma\left(\mathrm{Ca}_{12}\right)\left[\mathrm{s}^{-1}\right]$ is the ionisation rate of $\mathrm{Ca} \mathrm{I}$ by UV photons, and $C\left(\mathrm{Ca}_{12}\right)\left[\mathrm{cm}^{3} \mathrm{~s}^{-1}\right]$ is the collisional ionisation rate. Similar equations were used for other elements. The $\alpha_{\mathrm{rad}}$, $\alpha_{\text {die }}$ and $C$ parameters depend on the electron temperature $T_{\mathrm{e}}$.

The $\Gamma$ coefficients were adopted from the WJ2 model (de Boer et al. 1973). The recombination coefficients $\left(\alpha_{\text {rad }}\right.$ and $\left.\alpha_{\text {die }}\right)$ and the collisional ionisation rate coefficient $(C)$ for the $\mathrm{Ca}$ and Fe elements were taken from Shull \& van Steenberg (1982). For $\mathrm{K}$ and $\mathrm{Na}$ these parameters were adopted from Landini \& Monsignori Fossi (1991) and Landini \& Monsignori Fossi (1990). For Ti $\alpha_{\text {rad }}$ was taken from Badnell et al. (2006) and $\alpha_{\text {die }}$ comes from Mazzotta et al. (1998). We have used the Ti collisional ionisation rate coefficient $(C)$ from Voronov (1997).

The $\mathrm{CH}$ and $\mathrm{CN}$ molecules are not present in $\mathrm{CaFe}$ clouds (Bondar et al. 2007). The $\mathrm{CH}$ column density is directly proportional to the column density of the $\mathrm{H}_{2}$ molecule (e.g. Federman 1982; Danks et al. 1984; Gnaciński et al. 2007a). Furthermore, the $\mathrm{H}_{2}$ molecule is known to be formed on dust grains. We infer that the absence of simple molecules is caused by grains completely missing in $\mathrm{CaFe}$ clouds. Weingartner \& Draine (2001) have proposed recombinations on polycyclic aromatic hydrocarbons (PAHs) and on dust grains to play an important role in ion recombinations in cold neutral medium. Since we do not observe even the simplest molecules in $\mathrm{CaFe}$ clouds we do not consider recombinations on PAHs or dust grains here.

The second equation necessary to determine $N(\mathrm{Ca} \mathrm{I})$ and $N(\mathrm{Ca}$ II $)$ in our model is the conservation of the number of Ca atoms:

$N(\mathrm{Ca} \mathrm{I})+N(\mathrm{Ca} I \mathrm{II})+N(\mathrm{Ca} \mathrm{III})=N(\mathrm{H}) \times 10^{S S A(\mathrm{Ca})-12}$

where $N(\mathrm{Ca}$ III $)$ was calculated from the ionisation equilibrium with observed $N(\mathrm{Ca}$ II $)$. We have used the Solar System Abundances (SSA) as given by Grevesse \& Sauval (2000). Other elements (e.g. Fe) have the second ionisation potential larger than or close to $13.6 \mathrm{eV}$, and are present in the interstellar medium only in two ionisation stages:

$N\left(\mathrm{Fe}_{\mathrm{I}}\right)+N(\mathrm{Fe}$ II $)=N(\mathrm{H}) \times 10^{S S A(\mathrm{Fe})-12}$.

The hydrogen column density $N(\mathrm{H})$, the electron density $n_{\mathrm{e}}$ and the electron temperature $T_{\mathrm{e}}$ were treated as free parameters. We
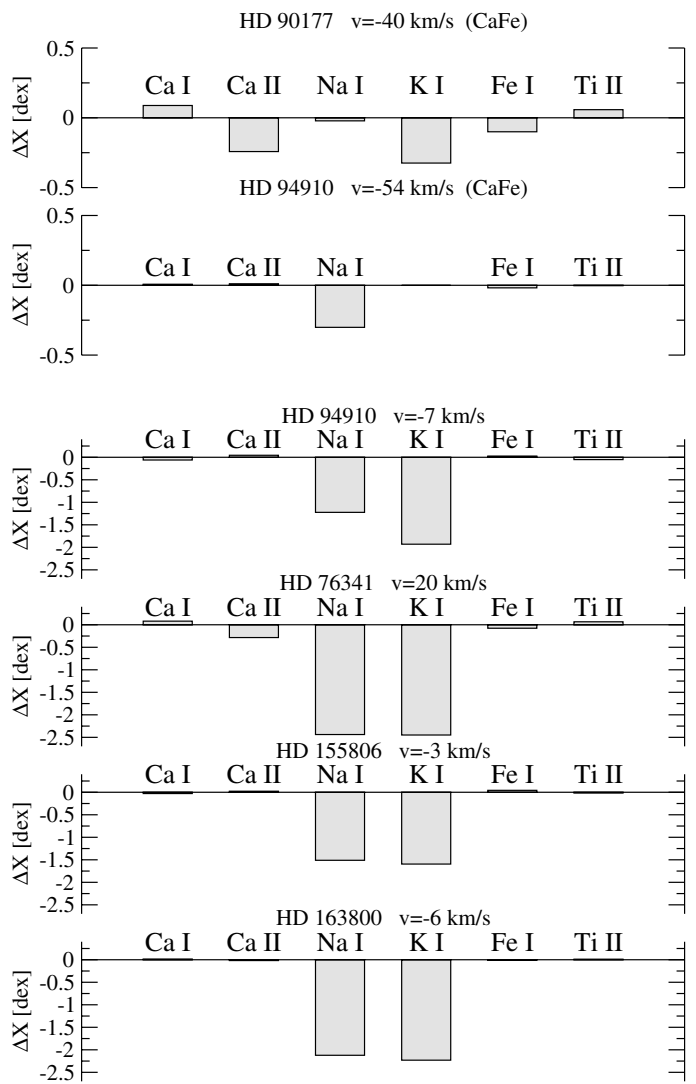

Fig. 3. The difference $\Delta X=\log N(X)_{\text {model }}-\log N(X)_{\text {obs }}$ between calculated column densities and the observed ones. Note the different $y$-axis scale for $\mathrm{CaFe}$ and non-CaFe clouds.

have used the "amoeba" procedure of Press et al. (1996) to find the minimum of the $\chi$ function:

$\chi^{2}=\sum_{X}\left(\frac{N(X)_{\text {model }}}{N(X)_{\text {obs }}}-1\right)^{2}$

where $X=\mathrm{Ca}$ I, Ca II, Na I, Fe I, K I and Ti II.

The model that best fits the observed column densities is presented in Table 2. $N(\mathrm{H})$ is the column density of hydrogen in a single analysed cloud. Physical conditions are described by the electron density $n_{\mathrm{e}}$ and the electron temperature $T_{\mathrm{e}}$. For comparison we also show the hydrogen column densities obtained from Ti II (assuming $N(\mathrm{Ti} \mathrm{II})=N(\mathrm{Ti})$ ) and from Ca II $\left(N(\mathrm{Ca})_{\text {obs }}+N(\mathrm{Ca} \text { II })_{\text {obs }}+N(\mathrm{Ca} \text { III })_{\text {model }}=N(\mathrm{Ca})\right)$ assuming Solar System abundances. The differences between the column densities obtained in our model and the observed ones $\Delta X=\log N(X)_{\text {model }}-\log N(X)_{\text {obs }}$ are shown in Fig. 3.

The errors of $N(\mathrm{H}), n_{\mathrm{e}}$ and $T_{\mathrm{e}}$ were calculated by modifying the column densities by the error presented in Table 1. First we added the errors to the column densities of ions and subtracted the error from the column densities of neutral elements. In a second run we added the error to the column densities of neutral species and subtracted the error from the ion column densities. In the case of interstellar clouds in the direction of HD 155806 and HD 163800 we could not achieve convergence in the second case.

\section{Discussion}

The absence of molecules in $\mathrm{CaFe}$ clouds implies that no dust is present in these clouds. This conclusion is confirmed by a 
Table 1. Column densities $\left(N\right.$ in $\left.\mathrm{cm}^{-2}\right)$ obtained from the UVES spectra.

\begin{tabular}{|c|c|c|c|c|c|c|c|}
\hline Star & $\begin{array}{c}v \\
{\left[\mathrm{~km} \mathrm{~s}^{-1}\right]}\end{array}$ & $\begin{array}{c}N(\text { Ca II }) 3970 \AA \\
\text { or } 3935 \AA\left[\mathrm{cm}^{-2}\right]\end{array}$ & $\begin{array}{c}N(\mathrm{Ca} \text { I) } 4228 \AA \\
{\left[\mathrm{cm}^{-2}\right]}\end{array}$ & $\begin{array}{c}N(\mathrm{~K} \mathrm{I}) 7701 \AA \\
\text { or } 7667 \AA\left[\mathrm{cm}^{-2}\right]\end{array}$ & $\begin{array}{c}N(\mathrm{Na} \mathrm{I}) 5892 \AA \\
\text { and } 5898 \AA\left[\mathrm{cm}^{-2}\right]\end{array}$ & $\begin{array}{c}N(\mathrm{Fe} \mathrm{I}) 3721 \AA \\
\text { or } 3861 \AA\left[\mathrm{cm}^{-2}\right]\end{array}$ & $\begin{array}{c}N(\text { Ti II }) 3385 \AA \\
\text { or } 3074 \AA\left[\mathrm{cm}^{-2}\right]\end{array}$ \\
\hline HD 90177 & $-40^{*}$ & $6.2 \mathrm{e} 12 \pm 7.8 \mathrm{e} 11$ & $5.6 \mathrm{e} 10 \pm 4.7 \mathrm{e} 09$ & $2.2 \mathrm{e} 10 \pm 8.5 \mathrm{e} 09$ & $1.3 \mathrm{e} 12 \pm 1.3 \mathrm{e} 10$ & $3.0 \mathrm{e} 12 \pm 1.6 \mathrm{e} 11$ & $6.5 \mathrm{e} 11 \pm 1.1 \mathrm{e} 11$ \\
\hline HD 9 & $-54^{*}$ & $\mathrm{e} 10$ & $6 \mathrm{e} 09$ & $<7 \mathrm{e} 09$ & & & 10 \\
\hline HD 94910 & -7 & $1.1 \mathrm{e} 12 \pm 1.6 \mathrm{e} 11$ & $5.0 \mathrm{e} 09 \pm 8.7 \mathrm{e} 08$ & $1.5 \mathrm{e} 11 \pm 1.1 \mathrm{e} 10$ & $3.5 \mathrm{e} 12 \pm 8.1 \mathrm{e} 10$ & $3.2 \mathrm{e} 11 \pm 1.0 \mathrm{e} 11$ & $1.2 \mathrm{e} 12 \pm 1.3 \mathrm{e} 11$ \\
\hline HD 76341 & 20 & $1.4 \mathrm{e} 12 \pm 4.0 \mathrm{e} 10$ & $5.6 \mathrm{e} 09 \pm 1.6 \mathrm{e} 09$ & $6.2 \mathrm{e} 11 \pm 1.4 \mathrm{e} 10$ & $7.2 \mathrm{e} 13 \pm 1.0 \mathrm{e} 12$ & $8.2 \mathrm{e} 11 \pm 7.8 \mathrm{e} 10$ & $2.8 \mathrm{e} 10$ \\
\hline HD 155806 & -3 & $3.4 \mathrm{e} 12 \pm 1.0 \mathrm{e} 11$ & $1.1 \mathrm{e} 10 \pm 4.4 \mathrm{e} 09$ & $2.4 \mathrm{e} 11 \pm 6.6 \mathrm{e} 09$ & $2.4 \mathrm{e} 13 \pm 1.7 \mathrm{e} 12$ & $1.6 \mathrm{e} 11 \pm 1.9 \mathrm{e} 10$ & $6.3 \mathrm{e} 11 \pm 4.6 \mathrm{e} 10$ \\
\hline HD 163800 & -6 & $2.4 \mathrm{e} 12 \pm 3.1 \mathrm{e} 11$ & $2.5 \mathrm{e} 10 \pm 1.9 \mathrm{e} 09$ & $6.7 \mathrm{e} 11 \pm 7.3 \mathrm{e} 09$ & $6.2 \mathrm{e} 13 \pm 7.5 \mathrm{e} 11$ & $1.4 \mathrm{e} 12 \pm 6.8 \mathrm{e} 10$ & $8.3 \mathrm{e} 11 \pm 5.0 \mathrm{e} 10$ \\
\hline
\end{tabular}

* $\mathrm{CaFe}$ cloud.

Table 2. Ionization equilibrium models.

\begin{tabular}{lllllll}
\hline \hline Star & HD 90177 & HD 94910 & HD 94910 & HD 76341 & HD 155806 & HD 163800 \\
$v\left[\mathrm{~km} \mathrm{~s}^{-1}\right]$ & $-40^{*}$ & $-54^{*}$ & -7 & 20 & -3 & -6 \\
\hline$N(\mathrm{H})\left[\mathrm{cm}^{-2}\right]$ & $1.5_{-0.2}^{+0.2} \times 10^{19}$ & $2.1_{-0.6}^{+0.4} \times 10^{18}$ & $1.2_{-0.1}^{+0.2} \times 10^{19}$ & $7.9_{-0.4}^{+0.3} \times 10^{18}$ & $1.1^{+0.1} \times 10^{19}$ & $1.2^{+0.1} \times 10^{19}$ \\
$n_{\mathrm{e}}\left[\mathrm{cm}^{-3}\right]$ & $1.03_{-0.05}^{+0.08}$ & $0.99_{-0.21}^{+0.46}$ & $0.20_{-0.01}^{+0.01}$ & $0.43_{-0.02}^{+0.03}$ & $0.60_{-0.12}$ & $0.50_{-0.01}$ \\
$T_{\mathrm{e}}[\mathrm{K}]$ & $8400_{-150}^{+300}$ & $10500_{-120}^{+600}$ & $8200_{-700}^{+600}$ & $8900_{-200}^{+200}$ & $5650_{-500}$ & $9000_{-300}$ \\
\hline$N(\mathrm{H})$ from Ca $\left[\mathrm{cm}^{-2}\right]$ & $1.6 \times 10^{19}$ & $2.2 \times 10^{18}$ & $1.2 \times 10^{19}$ & $8.2 \times 10^{18}$ & $1.0 \times 10^{19}$ & $1.2 \times 10^{19}$ \\
$N(\mathrm{H})$ from Ti $\left[\mathrm{cm}^{-2}\right]$ & $6.2 \times 10^{18}$ & $1.2 \times 10^{18}$ & $1.1 \times 10^{19}$ & $4.7 \times 10^{18}$ & - & $7.9 \times 10^{18}$ \\
\hline
\end{tabular}

${ }^{*} \mathrm{CaFe}$ cloud.

comparison of element abundances in $\mathrm{CaFe}$ clouds and in our model. In $\mathrm{CaFe}$ clouds elements with low condensation temperatures like $\mathrm{Na}$ or $\mathrm{K}\left(T_{\mathrm{C}} \approx 1000 \mathrm{~K}\right)$ do not show more depletion than the elements $\mathrm{Ca}, \mathrm{Fe}$ and $\mathrm{Ti}$ with higher condensation tempeature $\left(T_{\mathrm{C}}=1337 \div 1634 \mathrm{~K}\right)$. In fact the observed column densities of $\mathrm{NaI}$ and $\mathrm{KI}$ are even larger than the column densities derived from the best-fit model (Fig. 3). The depletion of atoms due to condensation onto dust grains is typically -0.2 to $-1.5 \mathrm{dex}$ (Gnaciński \& Krogulec 2006), while the highest deficit of observed abundance in our data is $0.088 \mathrm{dex}$ for $\mathrm{Ca} \mathrm{I}$ in the $\mathrm{CaFe}$ cloud to HD 90177. Therefore we conclude that the CaFe clouds have a composition similar to the Solar System abundances and no depletion onto dust grains is seen in the $\mathrm{CaFe}$ clouds.

For clouds analysed by Gnaciński et al. (2007b) the electron temperatures $T_{\mathrm{e}}$ are in the range $6800-11100 \mathrm{~K}$. The electron temperatures presented in Table 2 do not differ from these temperatures. Such temperatues are typical of warm clouds. For example Piskunov et al. (1997) found $T=5400-12700 \mathrm{~K}$ in 15 LISM clouds.

Both CaFe clouds analysed here have a high electron density $n_{\mathrm{e}} \approx 1 \mathrm{~cm}^{-3}$. In clouds analysed by Gnaciński et al. (2007b) the electron density is in the range $0.01-2.5 \mathrm{~cm}^{-3}$. The electron densities in the $\mathrm{CaFe}$ clouds are higher than electron densities in all 11 sight lines presented by Black \& van Dishoeck (1991).

Our model fails to fit $\mathrm{NaI}$ and $\mathrm{KI}$ in ordinary (non $\mathrm{CaFe}$ ) clouds. The observed column densities of $\mathrm{Na}$ I and $\mathrm{K}$ I are always higher by $1-3$ orders of magnitude than the theoretical ones. It is possible that a single set $\left(n_{\mathrm{e}}, T_{\mathrm{e}}\right)$ cannot describe the conditions in a non-CaFe cloud, because in the cloud's core the ionising photons are shielded by dust, and the conditions in the cloud's core differ substantially from those on the cloud surface. The $\mathrm{CaFe}$ clouds seem to be very homogenous in comparison to ordinary clouds, since we can fit the observed column densities with a single set of $n_{\mathrm{e}}$ and $T_{\mathrm{e}}$. In CaFe clouds we do not detect $\mathrm{CH}$ and $\mathrm{CH}+$ molecules (Bondar et al. 2007). The fact that we do not observe molecules in $\mathrm{CaFe}$ clouds supports the thesis that there are no cold cores in such clouds.

\section{Conclusions}

The results can be recapitulated as follows:

1. The CaFe interstellar clouds have a composition similar to the Solar System element abundance.

2. There is no depletion of elements onto dust grains in $\mathrm{CaFe}$ clouds.

3. The electron density $n_{\mathrm{e}}$ is high in CaFe clouds $\left(n_{\mathrm{e}} \approx 1 \mathrm{~cm}^{-3}\right)$.

4. The $\mathrm{CaFe}$ clouds seems to be thin clouds totally penetrated by UV radiation.

Acknowledgements. This research was based on spectra obtained by the UVES Paranal Observatory Project (ESO DDT Program ID 266.D-5655, see Bagnulo et al. 2003). We acknowledge the financial support from the University of Gdańsk (grant BW/5400-5-0305-7).

\section{References}

Badnell, N. R. 2006, ApJS, 167, 334

Bagnulo, S., Jehin, E., Ledoux, C., et al. 2003, Messenger, 114, 10

Black, J. H., \& van Dishoeck, E. F. 1991, ApJ, 369, L9

Bondar, A., Kozak, M., Gnaciński, P., et al. 2007, MNRAS, 378, 893

de Boer, K. S., Koppenaal, K., \& Pottasch, S. R. 1973, A\&A, 28, 145

Danks, A. C., Federman, S. R., \& Lambert, D. L. 1984, A\&A, 130, 62

Federman, S. R. 1982, ApJ, 257, 125

Hunter, I., Smoker, J. V., \& Keenan, F. P., et al. 2006, MNRAS, 367, 1478

Gnaciński, P., \& Krogulec, M. 2006, Acta Astron., 56, 373

Gnaciński, P., Krogulec, M., \& Krełowski, J. 2007a, Proc. Conf. Molecules in Space and Laboratory, Paris 14-18 May 2007

Gnaciński, P., Sikorski, J. K., \& Galazutdinov, G. A. 2007b, A\&A, 469 , 201

Grevesse, N., \& Sauval, A. J. 2000, Urania, 690, 248

Landini, M., \& Monsignori Fossi, B. C. 1990, A\&AS, 82, 229

Landini, M., \& Monsignori Fossi, B. C. 1991, A\&AS, 91, 183

Mazzotta, P., Mazzitelli, G., Colafrancesco, S., \& Vittorio, N. 1998, A\&AS, 133, 403

Morton, D. C. 2003, ApJS, 149, 205

Piskunov, N., Wood, B. E., Linsky, J. L., Dempsey, R. C., \& Ayres, T. R. 1997, ApJ, 474, 315

Press, W. H., Teukolsky, S. A., Vatterling, W. T., \& Flannery, B. P. 1996, Numerical Recipes in Fortran 90, Cambridge University Press

Shull, J. M., \& van Steenberg, M. 1982, ApJS, 48, 95

Voronov, G. S. 1997, Atomic Data and Nuclear Data Tables, 65, 1

Weingartner, J. C., \& Draine, B. T. 2001, ApJ, 563, 842 